\title{
STRESS CORRELATION AND TEMPOROMANDIBULAR DISORDER IN UNIVERSITY STUDENTS
}

R. Riffel ${ }^{1}$, C. Riffel- $\mathrm{CDT}^{2}$, M. Fernandes- $\mathrm{MS}^{3}$, V. Pietrobelli- VL ${ }^{4}$, R. Nascimento- RM4, B. Tams- BD ${ }^{1}$, F. Rhoden- $\mathrm{FK}^{2}$, I. Leão- $\mathrm{IF}^{2}$

Objectives: Correlating Emotional Stress and TMD in College Students

Background: Stress is defined as general syndrome of adaptation (GSA), whose purpose is the return to homeostasis, and thus, secondary to a constant stimulus and potentially generating emotional imbalance. Chronic pain is one of the main causes of physical, psychosocial and labor losses. Temporomandibular dysfunction (TMD) is among the most common causes of chronic pain, reaching between $31 \%$ and $61 \%$ of the population. Its etiology is multifactorial with stress being a predisposing factor.

Table 1- Spearman correlation between the TMD-F and SRRS data, for all sampling TMD-F X SRRS R P $0,217 \quad 0,0001$

Materials and Methods: The studied population was composed of university students from Universidade de Passo Fundo, Brazil. Questionnaires were used to identify TMD and stress signs in 696 students. The results were tabulated and analyzed by means of descriptive statistics, chi-square test and Spearman's correlation.

${ }^{1}$ UFFS, Psychiatry, Passo fundo, Brazil;

${ }^{2}$ FASURGS, Orthodontics, Passo fundo, Brazil;

${ }^{3}$ UFFS, Pharmacology, Passo fundo, Brazil;

${ }^{4}$ UFFS, Academic, Passo fundo, Brazil.

\begin{tabular}{|ccccc|}
\hline Table 2 - Results & Chi-square test of TMD levels intersect with SRRS \\
SRRS & TMD 1 & $\begin{array}{c}\text { TMD-F } \\
\text { TMD 2 }\end{array}$ & TMD3 & P \\
Very low & 86 & 28 & 11 & \\
Low & 35 & 13 & 6 & 0,3703 \\
Mild & 64 & 30 & 10 & \\
High & 124 & 69 & 13 & \\
Total & 309 & 140 & 40 & \\
\hline
\end{tabular}

\begin{tabular}{ccccccc} 
Table 3 - Distribution of TMD-F and SRRS degrees in the studied populat \\
TMD-F & & Very low & Low & Mild & High & Total \\
Absent & $\mathrm{N}$ & 96 & 24 & 42 & 45 & 207 \\
& $\%$ & $46.4 \%$ & $11.6 \%$ & $20.3 \%$ & $21.7 \%$ & $100.0 \%$ \\
Low TMD & $\mathrm{N}$ & 86 & 35 & 64 & 124 & 309 \\
& $\%$ & $27.8 \%$ & $11.3 \%$ & $20.7 \%$ & $40.1 \%$ & $100.0 \%$ \\
Mild TMD & $\mathrm{N}$ & 28 & 13 & 30 & 69 & 140 \\
& $\%$ & $20.0 \%$ & $9.3 \%$ & $21.4 \%$ & $49.3 \%$ & $100.0 \%$ \\
Severe TMD & $\%$ & 11 & 6 & 10 & 13 & 40 \\
Total & $\mathrm{N}$ & $27.5 \%$ & $15.0 \%$ & $25.0 \%$ & $32.5 \%$ & $100.0 \%$ \\
& $\mathrm{~N}$ & 221 & 78 & 146 & 251 & 696 \\
& & $31.8 \%$ & $11.2 \%$ & $21.0 \%$ & $36.1 \%$ & $100.0 \%$ \\
\hline
\end{tabular}

Results and Conclusions: Evaluating the DTM level correlated to stress level, there was a weak positive correlation (Table 1). Crossing the TMD grades and the stress levels, there was no statistical difference in the results $(p=0.3703)$ (table 2). Analyzing levels of DTM-F and stress, it was observed that the mild TMD ( $N=309,44.4 \%)$ and high stress ( $\mathrm{N}=251,36.1 \%$ ) were the most frequent (Table 3). There was a higher prevalence of higher levels of stress (63.07\%) and TMD $(70.76 \%)$ in women. It was weak correlation between TMD and stress was found in the groups. Correlating stress in TMD patients, we observed more intense and frequent signs than in non-carriers. There is a low percentage of individuals who are free of TMD. 